\title{
PERFORMANCE EVALUATION OF MULTIPLE TRANSFORM WATERMARKING SYSTEM FOR PRIVACY PROTECTION OF MEDICAL DATA USING PSNR AND NC
}

\author{
Mutiu Adesina ADEGBOYE*, Lukman Adewale AJAO**, Taofeeq Alabi BADMUS* \\ *Department of Computer Engineering, Faculty Engineering, Federal University Oye-Ekiti, Ekiti State, Nigeria, tel. \\ +2347038117823, e-mail: mutiu.adegboye@fuoye.edu.ng \\ ${ }^{* *}$ Department of Computer Engineering, Federal University of Technology, Minna, Niger State Nigeria
}

\begin{abstract}
This paper presents the performance evaluation of a developed multiple transform watermarking system for privacy protection of medical data using Peak Signal to Noise Ratio (PSNR) and Normalized Cross-Correlation (NC). The PSNR was used to evaluate the imperceptibility of the system, while the NC was used to evaluate the robustness of the system under the various attacks include: Gaussian noise, pepper and salt noise, sharp enhancing, image cutting, image compression, low pass filter and image rotation. The obtained result showed that the similarity between original image and watermarked image has PSNR of 52.4595dB as compared to the existing system of $50.0285 \mathrm{~dB}$. This indicates that the proposed scheme can conceal the watermark better, and as well retains the image quality.
\end{abstract}

Keywords: Arnold transform; Discrete cosine transform; Discrete wavelet transform; Medical Data; Watermarking

\section{INTRODUCTION}

In today's society, advance in technology has opened new potentials to healthcare and medicine practice. This enables the use of Information and Communication Technologies (ICTs) in the health sector for carrying out diagnosis and consultation regardless of location of the patient and healthcare practitioners [1], as well as administrative and educational purposes [2]. Owing to the sensitive nature of patient's data, it is imperative to prevent unauthorized access to the patient's medical information. The health practitioner who issues and authenticates the medical data should ensure its confidentiality as unacceptable tampering of the data might result in misdiagnosis. Therefore, security problem becomes an essential issue that requires urgent solution.

Encryption has been widely used for securing data [3]. With the use of encryption certain security measure has been accomplished, it makes the secrete messages incomprehensible and unnatural. However, the use of encryption usually attracts some unpremeditated attention of observers [4]. This necessitates new security techniques such as steganography and watermarking. Steganography is used for secrete communication, whereas watermarking is used for content protection, copyright management, content authentication and tamper detection [5]. The term watermark originated from the German called Watermark [6]. watermarking is defined as the direct method of embedding additional information into original content or host signal [7]. The classifications of watermarking can be based on type of watermark, domain type and type of information requires in the extraction or detection process.

Pseudo Random Number (PRN) and visual watermark are the common types of watermark. For the PRN, sequence can be generated through the feeding of signal generator with a secrete seed, while in visual watermark, the watermark can be reconstructed, and its visual quality can be evaluated. The classification of watermark based on information type is grouped into three categories namely; blind system, semi-blind system and non-blind system [8]. Both blind system and semi-blind systems are usually less robust, leading it to high rate of false negative (not detecting the watermark in watermark image) and false positive (detecting the watermark in not watermark image).

In domain type watermark classification, the method of embedding watermark are pixel domain and domain transform. For the pixel domain method, watermark is embedded through the modification of the pixel values of the original image. This method is practically simple, it requires very little computation cost both at the watermark encoder and decoder. However, it is not dependable when subjected to media operation such as low compression or filtering. This makes it less robust to various attacks [9]. Similarly, watermark can be embedded through the modification of transform coefficient in transform domain. The strength of this technique is based on its ability to take advantage of special properties of domain alternation to address the limitation of pixel based technique [9]. Discrete Wavelet Transform (DWT), Discrete Cosine Transform (DCT) and Discrete Fourier Transform (DFT) are the three main techniques of transforming the data in frequency domain [10].

The DCT, DWT and DFT have been used in the literature for the digital watermarking. In effort to make the system to be more robust for the medical data. This paper proposed a modified multiple transform system using DWT, DCT and Arnold transform. The idea of combining these transforms is based on the fact that the combination could give better performance.

\section{THE REVIEW OF THE RELATED WORK}

Several studies have been conducted on watermarking. However, most of the prior researches were based on DWT, DCT and combination of both. In the approach of [11], watermark insertion and extraction proposed using DWT proposed. The watermark recovered proved significant in comparison with original image. A similar multiple nonblind watermarking system using DWT proposed by [12]. The DWT applied to the gray scale image through the modification of lowest frequency (LL) and highest frequency $(\mathrm{HH})$ sub-band coefficient. The obtained result 
shows high degree of robustness and fidelity against a large range of attacks.

An optimized image watermarking approach using Differential Equation (DE) in DWT and Singular Value Decomposition (SDV) domain proposed by [13]. The outcome of their finding shows significant performance under various attacks. The proposed system can retain image quality and transparency digital watermark. However, its computational cost is high. [14] proposed a robust and secure image watermarking scheme through the embedding of watermark in the de-interlace images using wavelet transform. The system demonstrated good robustness against various attacks. Comparison of the digital image watermarking technique using only DWT and Combination of the DWT and DCT proposed in the approach of [15]. The performance of the proposed system was evaluated using peak signal (PSNR). The experimental result shows that combination of the transforms performed better than single transform.

A similar digital watermarking algorithm using combination of DCT and DWT proposed by [16]. The performance of the algorithm was evaluated using gray level image size of $255^{*} 256$ as a host image for embedded watermark, and a binary image size of $32 * 32$ logo as watermark image. The simulation result demonstrates that the scheme performance appeared better and also robust against many attacks such as salt noise, filter, sharp enhancement, cutting image edge rotation and compression.

\subsection{The DWT and DCT}

The DWT and DCT have been widely used in the literature for many applications. In this section, the relevance of the two transforms to the implementation of watermarking is briefly discussed.

\subsubsection{The DWT}

The DWT is a modern method recently applied in image compression, image processing and watermarking. The time-frequency representation of signal can be accomplished with the use of DWT. The concept of DCT is based on minor waves, well known as wavelet. For a 2D image, if DWT equivalent applied to processing the image using 2-D filters in each of the image dimensions, the filters will partition the image into four non-overlapping multi-resolution sub-bands in which one is represented by Lowest Bands (LL) and the other high band by LH, HL and $\mathrm{HH}$ respectively. Fig. 1 illustrates a 2-dimensional image after the 3 times DWT decomposition [17]. The symbol $\mathrm{H}$ represents high pass filter while $\mathrm{L}$ represents the low pass filter. The contents of low frequency band contain information near to input image, while the contents of high frequency band level contain the aspect of the image. The DWT is a multi-resolution with a great spatial localization. This makes it widely used for image watermarking.

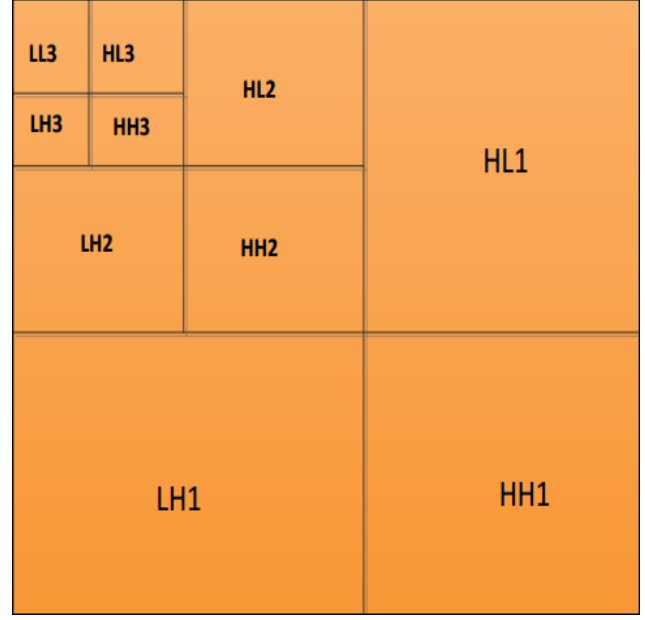

Fig. 1 Sketch map of image DWT decomposed

\subsubsection{Discrete Cosine Transform}

The DCT is a frequently used method for the transformation of a signal into basic frequency component [18]. In DCT transform, an image is represented as a summation of sinusoids of varying frequencies and magnitudes Owing to it excellent capability of energy compression and decorrelation, it is faster than DFT due to the fact that its transform kernel is a complex exponential [19]. With an input image $f(x, y)$ of a two-dimensional matrix having $M \times N$ size, a DCT can be obtained using the equations (1), (2) and (3) respectively;

$$
C(0,0)=\sqrt[1]{M N} \sum_{x=0}^{M-1} \sum_{y=0}^{N-1} f(x, y)
$$

$C(u, v)=\sqrt[2]{M N} \sum_{x=0}^{M-1} \sum_{y=0}^{N-1} f(x, y) \cos \frac{(2 x+1) u \pi}{2 M}$

$\cos \frac{(2 y+1) v \pi}{2 N}$

Where: $u=1,2,3, \ldots M-1$

$$
v=1,2,3, \ldots N-1
$$

The inverse transform can be obtained using equation given by;

$$
\begin{aligned}
& C(u, v)=\sqrt[1]{M N}(0,0)+\sqrt[2]{M N} \sum_{u=0}^{M-1} \sum_{v=0}^{N-1} C(u, v) \cos \\
& \frac{(2 x+1) u \pi}{2 M} \cos \frac{(2 y+1) v \pi}{2 N}
\end{aligned}
$$

Where: $x=1,2,3, \ldots M-1$

$$
y=1,2,3, \ldots N-1
$$




\section{METHODS}

The materials and method use in the course of this research is presented in this section.

\subsection{Data acquirement}

A 2-Dimensional 256 × 256 medical record and magnetic resonance brain image were used as the original cover host image and watermark image respectively. The magnetic resonance brain image was obtained from the study of [20], while the medical record was acquired with the use of a digital camera. This module forms input to the system. Fig. 2. depicts the sample of the images.
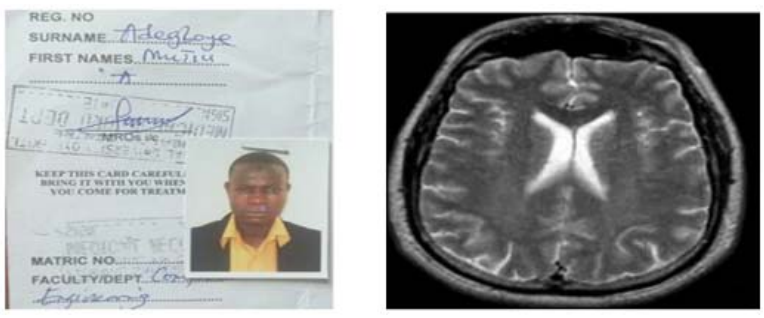

Fig. 2 Image for (a) Medical record and (b) Magnetic resonance brain

\subsection{Arnold transforms}

Arnold transform (AT) is an algorithm also called cat face transform [21]. It was applied in this study for scrambling the gray values layout of the images. The algorithm was introduced to prevent an attacker from detecting the watermark signal after the watermark processes. Even, if the algorithm was able to detect, a lot of test will be required to recover the original watermark. Given a squire digital image represented as a $N x N$ matrix, the image pixel scrambling can be achieved using equation (4);

$\left[\begin{array}{l}x^{\prime} \\ y^{\prime}\end{array}\right]=\left[\begin{array}{ll}1 & 1 \\ 1 & 2\end{array}\right] x\left[\begin{array}{l}x \\ y\end{array}\right]$

where: $(x, y)=$ coordinate location of the original image pixels, $\left(x^{\prime}, y^{\prime}\right)=$ coordinate location of the original image pixels after the transformation.

\subsection{Watermark Insertion Procedure}

The proposed watermarking scheme for the medical data is based on concept proposed by [16]. The watermarking processes were achieved through the application of 2-level DWT applied on host images. The DWT decomposed into four non-overlapping multiresolution. The proposed watermark was embedded in the middle frequency sub-band $\left.{ }_{H L x}\right)$ based on the fact that acceptable imperceptibility and robustness performance can be accurately achieved at this level [22], [23]. Fig. 3 illustrates the watermark insertion procedures followed by expiation of the steps involved.
The steps involved in the insertion process is as follows:

Step1: The DWT was applied to decompose the cover host image into four non-overlapping multi resolution subband as follows: $L L_{1}, H L_{1}, L H_{1}$ and $H H_{1}$

Step2: The DWT was further applied to divide $L L_{1}$ into sub-band and $\mathrm{LH}_{2}$ was chosen for the embedded watermark since it is at the middle frequency sub-band, imperceptibility and robustness performance can be efficiently achieved (Tsai \& H. Hung, 2005; Al-Haj, 2007).

Step3: The selected sub-band $\mathrm{LH}_{2}$ was decomposed into $4 \times 4$ blocks. This followed by application DCT applied to the each of the selected block of sub-band.

Step4: The Arnold transform algorithm performed to scramble the watermark signal, and the scrambling processes was repeated until best result attained.

Step5: The scramble watermark image was reformulated into a vector of zeros and ones.

Step6: Generated of uncorrelated pseudorandom sequences were with the aid of two key. One key was used for the insertion of the watermark bit0 (PIN_0), while the second key was used for the insertion of the watermark bit 1 (PIN_1).

Step7: The two pseudorandom sequences (PIN_0 and PIN 1) were embedded with a gain factor $(\alpha)$ in the $4 \times 4$ block DCT transformed of the selected DWT sub-band of the host images. By representing the mid-band matrix coefficient of the DCT transformed with X, the embedding process can be obtained using equation (5).

$X^{\prime}=\left\{\begin{array}{l}\mathrm{X}+\alpha^{\prime} \text { PIN_0, if watermarkbit is } 0 \\ \mathrm{X}+\alpha^{\prime} \text { PIN_1, if watermarkbit is } 1\end{array}\right.$

Step8: After the mid-band coefficients have been modified to embed the watermark bits as described in previous steps, an Inverse DCT (IDCT) was performed on each of the blocks.

Step9: IDWT was executed on the DWT transformed image to produce the watermarked host image.

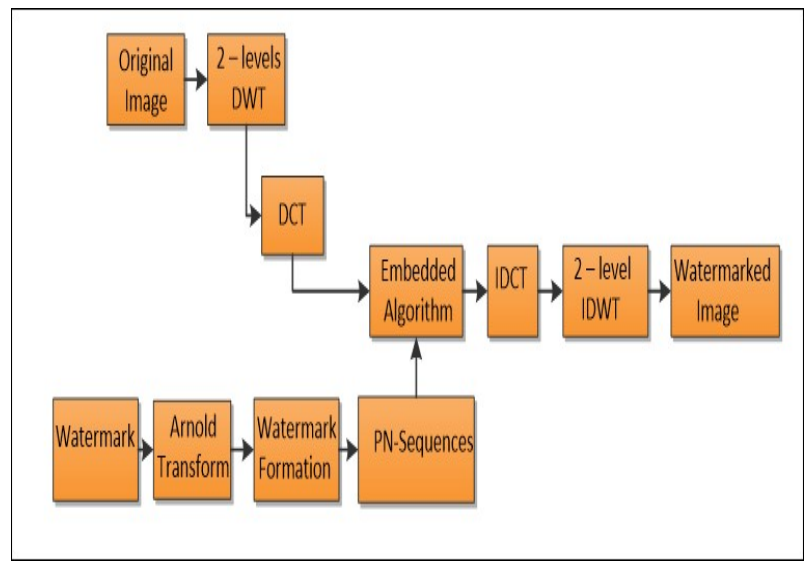

Fig. 3 Combined DWT, DCT and AT watermark insertion procedures 


\subsection{Watermark Extraction Procedure}

Extraction watermark algorithm is a blind watermarking algorithm, therefore original host image is not required for the watermark extraction processes. The procedure for watermark extraction is exemplified in Fig.4. Follows by description of the steps undergo.

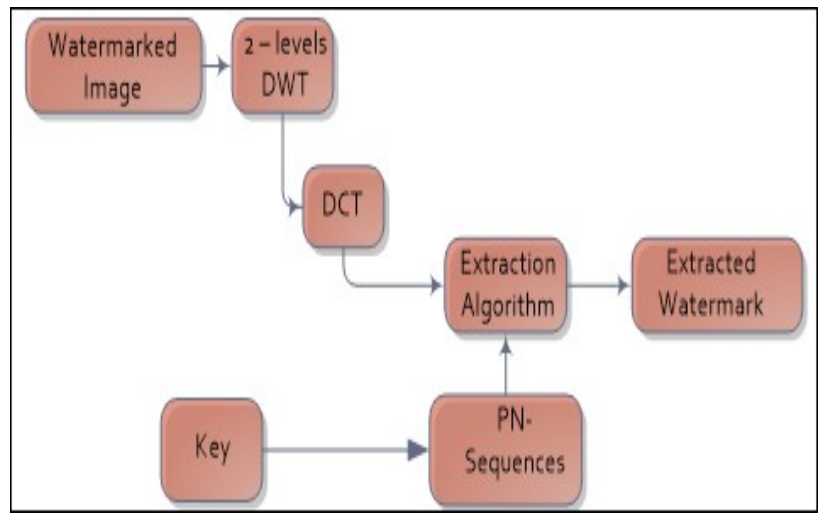

Fig. 4 Combined DWT - DCT watermark extraction procedures

The extraction procedure is similar to insertion algorithm. The steps involved in extraction process is as follows:

Step1: The DWT applied to watermarked image to decompose it into four non-overlapping multi-resolution sub-bands as follows: $L L_{1}, H L_{1}, L H_{1}$ and $H H_{1}$

Step2: The DWT was further applied to $L L_{1}$ the obtain the four smaller sub-bands, and sub-band $\mathrm{LH}_{2}$ was selected.

Step3: The $\mathrm{LH}_{2}$ was chosen and decomposed into $4 \mathrm{x}$ 4 block. Followed by DCT applied to each of the selected sub-band blocks, and mid-band coefficients of DCT transformed the block.

Step4: The two pseudorandom sequences were regenerated using the same key used for the watermark insertion.

Step5: The correlation between the mid-band coefficient and the two generated pseudorandom sequences (PIN_0 and PIN_1) were compared with the each of the block in the sub-band $\mathrm{LH}_{2}$ The correlation computation was based on the condition that; if the correlation between the PIN_0 higher than the correlation between the PIN_1, then the extracted watermarked bit is 0 , else the extracted watermark is 1 .

Step6: The watermark was reconstructed using the extracted watermark bits, and end by computation of the similarity between the original and extracted watermarks.

\subsection{System Performance Evaluation}

In evaluating the performance of the proposed scheme, imperceptibility and robustness were the metrics considered. It was chosen based on the fact that they are the metrics commonly evaluated in testing performance of the watermark algorithm [24], [25]. The description of the metrics is as follows:

\subsubsection{Imperceptibility}

Imperceptibility means inability to distinguish between watermark image and original image by human eyes. Peak Signal to Noise Ratio (PSNR) was used to evaluate the imperceptibility of the developed algorithm. Considering a $m \times n$ grayscale image, the PSNR can be defined with the equation given in (6).

$$
P S N R=10 \times \log _{10} \frac{255 \times 255}{\sum_{i=1}^{m} \sum_{j=1}^{n}\left(P_{i j}-q_{i j}\right)}(d B)
$$

Where $P_{i j}$ represents the pixel values in the row $i$ and $j$ of the host image and $q_{i j}$ represents the pixel values in the row $i$ and $j$ of the embedded image.

\subsubsection{Robustness}

The inability of an unauthorized user or attacker to eliminate the watermark from the embedded data. Normalized Correlation Coefficient (NC) was used to evaluate robustness the proposed scheme is referred to as robustness. Considering the grayscale image of a $m \times n$ matrix, the $\mathrm{NC}$ can be determine using equation given in (7).

$$
N C=\frac{\sum_{i=1}^{m} \sum_{j=1}^{n} P_{i j} q_{i j}}{\sum_{i=1}^{m} \sum_{j=1}^{n} P_{i j}{ }^{2}}
$$

Where $P_{i j}$ represents the pixel values in the row $i$ and $j$ of the host image and $q_{i j}$ represents the pixel values in the row $i$ and $j$ of the embedded image.

\section{SIMULATION RESULTS}

The proposed watermark scheme was experimented as described in Section 3.0. A $256 \times 256$ medical record is used as the original cover host image, and a $128 \times 128$ magnetic resonance brain image as a watermark image. The original cover host image and watermarked image are shown in Fig. 5 (a) and (b) respectively. By observing the results shown in Fig. 5 (a) and (b), it can be seen that the similarity between the original image and watermark image cannot be visually distinguished by human eyes. Similarly, the similarity between the original image and watermark image was evaluated using PSNR. The proposed scheme has PSN of $50.4595 \mathrm{~dB}$ as compared to [16] of $50.02850 \mathrm{~dB}$. This indicates that the proposed scheme conceals the watermarking excellently, and as well keeps the image quality well. 


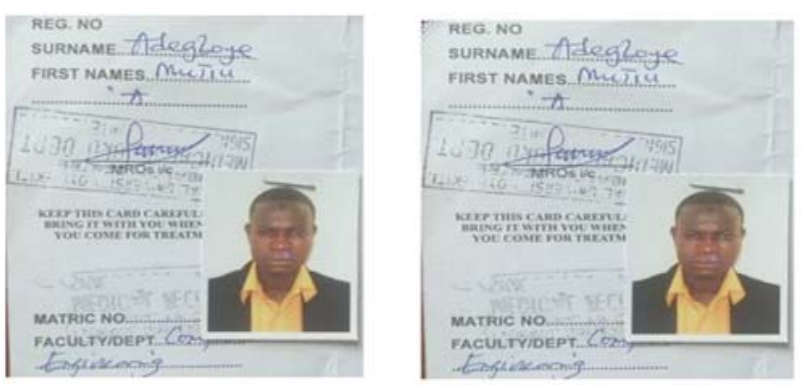

Fig. 5 Data illustration for (a) Original cover host image and (b) Watermarked image

In order to test the performance of the proposed scheme, the algorithm is tested under various attacks using MATLAB with: Gaussian noise, pepper and salt noise, sharp enhancement, image cutting, image compression, low-pass filter, rotation at 300 and 600 respectively. The simulation results under different attacks are shown in Fig. 6 , while the extracted watermarks from the attacked images are demonstrated in Fig. 7.

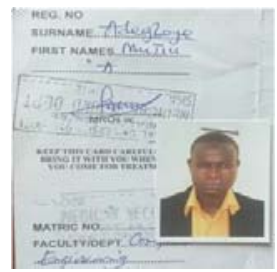

(a)

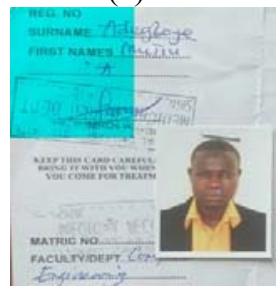

(d)

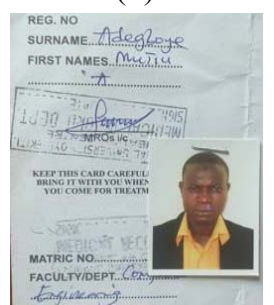

(g)

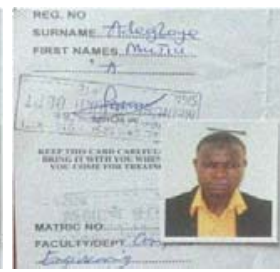

(b)

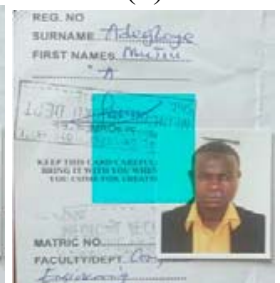

(e)

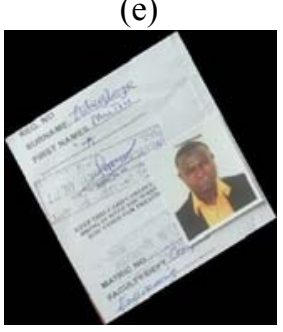

(h)

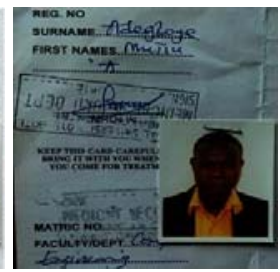

(c)

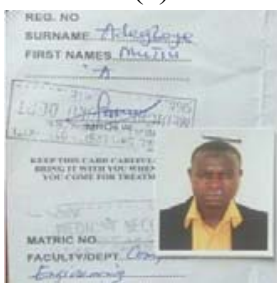

(f)

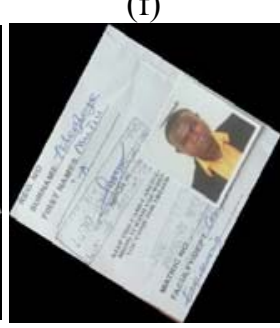

(i)
Fig. 6 Images with attacks for (a) Gaussian noise, (b) Pepper and salt noise (c), Sharp enhancing, (d) Image cutting (1/4 edge), (e) Image cutting (1/4 center), (f) Low-pass filter, (g) Image rotation (300) and (h) Image rotation (600)

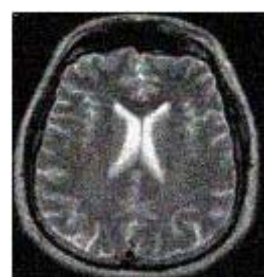

(a)

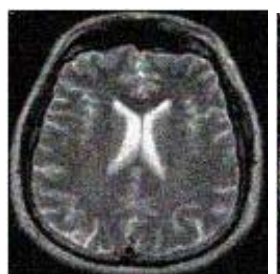

(b)

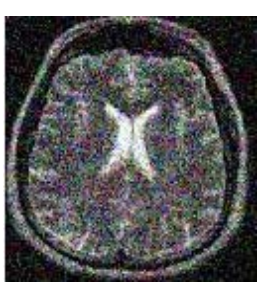

(c)

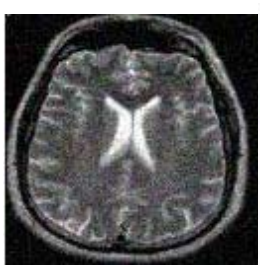

(d)

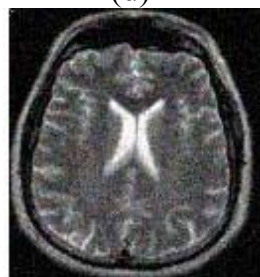

(g)

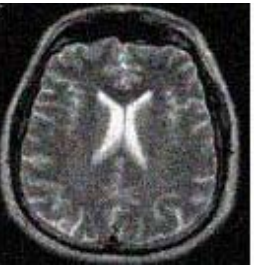

(e)

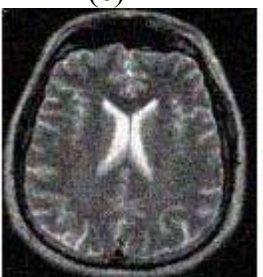

(h)

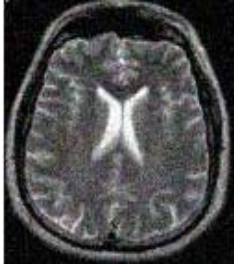

(f)

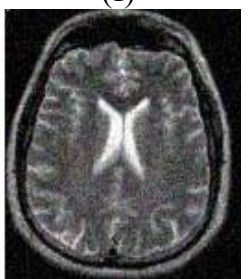

(i)
Fig. 7 Extracted watermarks from attacked images

The imperceptibility and robustness of the proposed algorithm was tested and evaluated under the various attacks as shown in Fig. 6. The PSNR values and NC values obtained is shown in Table 1.

Table 1 PSNR and NC values under various watermarked attacks

\begin{tabular}{|c|c|c|}
\hline Noise Type & PSNR $(\mathrm{dB})$ & $\mathrm{NC}$ \\
\hline Gaussian noise & 29.2835 & 0.8900 \\
\hline Pepper and sail noise & 30.5449 & 0.8958 \\
\hline Sharp enhancing & 35.0453 & 0.9101 \\
\hline $\begin{array}{c}\text { Image cutting }(1 / 4 \\
\text { edge) }\end{array}$ & 31.8645 & 0.8159 \\
\hline $\begin{array}{c}\text { Image cutting }(1 / 4 \\
\text { center })\end{array}$ & 32.9733 & 0.8079 \\
\hline Low-pass filter & 37.6281 & 0.9316 \\
\hline Image rotation $\left(30^{0}\right)$ & 26.5269 & 0.7603 \\
\hline Image rotation $\left(60^{\circ}\right)$ & 25.8135 & 0.7515 \\
\hline
\end{tabular}

Since our algorithm is based on the existing scheme proposed by [16], therefore, Table 2 compared obtained results with the results of Jiansheng et al. [16]. Fig. 7 shows the comparative plot between the existing system and the proposed scheme.

As shown in Fig. 7, it can be seen that inclusion of the Arnold transform gives better performance than only DWT-DCT. The maximum difference of $5.0266 \mathrm{~dB}$ obtained between the proposed and existing system under the image rotation attacked, while the minimum PSNR value of $1.1984 \mathrm{bB}$ obtained under the Gaussian noise attacked. Similarly, the proposed scheme gives maximum value of $0.9316 \mathrm{NC}$ under the low pass filter attacked as compare to the existing system of $0.9132 \mathrm{NC}$. This indicates that imperceptibility and robustness of the watermark algorithm can be improved with the inclusion of Arnold transform on DWT-DCT watermark algorithm. 
Table 2 Performance comparison

\begin{tabular}{|c|c|c|c|c|}
\hline \multirow{2}{*}{ Image type under attack } & \multicolumn{2}{|c|}{ PSNR (dB) } & \multicolumn{2}{c|}{ NC } \\
\cline { 2 - 5 } & Existing system [16] & $\begin{array}{c}\text { Proposed } \\
\text { scheme }\end{array}$ & $\begin{array}{c}\text { Existing system } \\
{[16]}\end{array}$ & $\begin{array}{c}\text { Proposed } \\
\text { scheme }\end{array}$ \\
\hline Gaussian noise & 28.0851 & 29.2835 & 0.8643 & 0.8900 \\
\hline Pepper and salt noise & - & 30.5449 & - & 0.8958 \\
\hline Sharp enhancing & 32.0763 & 35.0453 & 0.8962 & 0.9101 \\
\hline Image compression & 31.7749 & 33.0391 & 0.8518 & 0.8853 \\
\hline Low-pass filter & 35.5801 & 37.6281 & 0.9132 & 0.9316 \\
\hline Image cutting (1/4 edge) & 27.3359 & 31.8645 & 0.7089 & 0.8159 \\
\hline Image cutting $(1 / 4$ center) & - & 32.9733 & - & 0.8077 \\
\hline Image rotation $\left(30^{\circ}\right)$ & 21.5003 & 26.5269 & 0.5034 & 0.7603 \\
\hline Image rotation $\left(60^{\circ}\right)$ & - & 25.8135 & - & 0.7515 \\
\hline
\end{tabular}

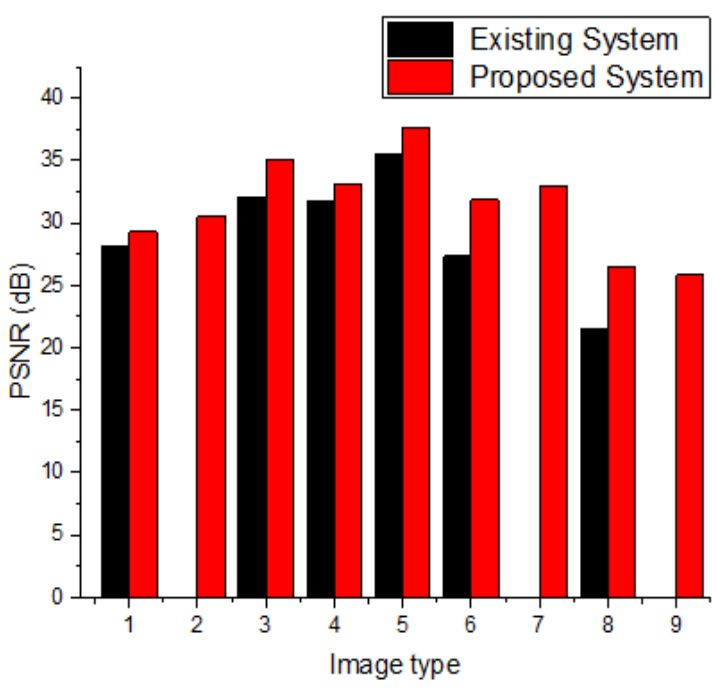

(a)

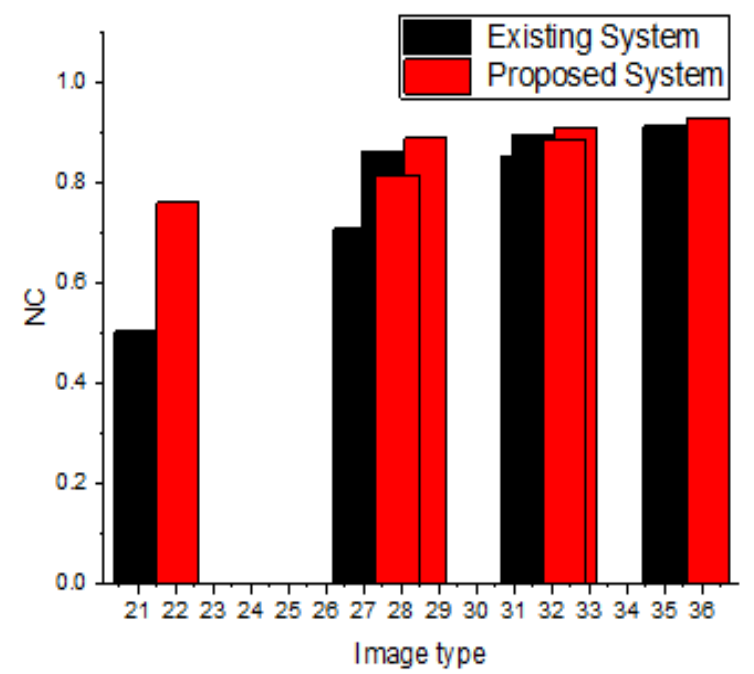

(b)

Fig. 7 Comparison plot of between the existing System and proposed scheme for the (a) PSNR and (b) NC

\section{CONCLUSIONS}

In this paper, a watermarking algorithm for privacy protection of the medical data using DWT, DCT and Arnold transform is implemented. The study has successful evaluate the performance of the proposed scheme under various attacks using MATLAB with: Gaussian noise, pepper and salt noise, sharp enhancement, image cutting, image compression, low-pass filter, rotation at 300 and 600 respectively. The performance of the system was evaluated in terms of imperceptibility and robustness using PSNR and NC. The obtained result shows desirable performance compare with the existing system based on DWT-DCT only. As a result, combining the DWT, DCT and Arnold transforms show better capacity to provide better imperceptibility and robustness against signal processing attacks. This study has proved the guaranty of protecting the privacy of medical data with the combination of the multiple transforms. However, other image scrambling methods such as; Gray code, Fass curve and magic square can be used.

\section{REFERENCES}

[1] ADEGBOYE, M. A. - OLANIYAN, O. M. - AJAO, L. A. - NNAMDI, O. S. - CANDIDUS, O. O.: Internet of things: survey of the security challenges and countermeasures, Journal of Advances in Scientific Research \& Applications, Vol. 3, pp. 97 105, Dec. 2016.

[2] OlANiYAN, O. M. - ADEGBOYE, M. A. OMODUNBI, B. - ESAN, A.: Electronic Health Technology and its Application Area: A Review. Advances in multidisciplinary \& Scientific Research Journal, Vol. 3, No. 1, pp. 125-130, 20017.

[3] PETITCOLAS, F. A. P.: Introduction to Information Hiding. In: KATZENBEISSER, S. - PETITCOLAS, F.A.P (ed.) (2000) Information hiding Techniques for Steganography and Digital Watermarking. Norwood: Artech House, INC., 2000. 
[4] SHEJUL, A. A. - KULKARNI, U. L.: A secure skin tone based steganography using wavelet transform," International Journal of Computer Theory and Engineering, vol. 3, no. 1, pp. 16-22, 2011.

[5] VIDYASAGAR, M. P. - SONG, H. - CHANG, E.: A survey of digital image watermarking techniques. $20053 r d$ IEEE International Conference on Industrial Informatics (INDIN), pp. 409- 416, 2005.

[6] RAWAT, N. - MANCHANDA, R.: Review of methodologies and techniques for digital watermarking. International Journal of Emerging Technology and Advanced Engineering, Vol. 4, No. 4, pp. 237-240, 2014.

[7] ADEGBOYEA, M. A. - AJAO, L. A. - BADMUS, T. A. - NNAMDI, O. S.: Multiple Transform Watertermarking System for Privacy Protection of Medical Data. 7th International conference on Mobile $e$-service, Adeleke Ede, Nigeria, $17^{\text {th }}$ to $19^{\text {th }}$ Oct., 2017.

[8] COX, I. - MILlER, M. - BLOOM, J.: Digital Watermarking, Academic Press, USA, 2002.

[9] PATEL, M. - SAJJA, P. S. - SHETH, R. K.: Analysis and survey of digital watermarking techniques. International Journal of Advanced Research in Computer Science and Software Engineering Research Paper, Vol. 3, No. 10, pp. 203-210, 2013.

[10] KAUR, M. - MALHOTRA, S.: Review paper on digital Image watermarking technique for robustness. International Journal of Advanced Research in Computer Science and Software Engineering, Vol. 4, No. 5, pp. 948-952, 2014.

[11] SHING, A. P. - MISHRA, A.: Wavelet based watermarking on digital image. Indian Journal of Computer Science and Engineering, Vol. 1, No. 2, pp. 86-91, 2011.

[12] OUAZZANE, H. - MAHERSIA, H. - HAMROUNI, K.: A Robust Multiple Watermarking Scheme based on the DWT. 10th IEEE International MultiConference on Systems, Signals and Devices(SSD), pp. 18-21, 2013.

[13] MUSRAT, A. - WOOK, A. - C. MILliE, P.: An optimized watermarking technique based on DE in DWT-SVD domain. IEEE Symposium on Differential Evolution, pp. 99-104, 2013.

[14] SRIDHAR, B. - ARUN, D. C.: On Secure Multiple Image Watermarking Techniques using DWT, IEEE20180, 2012.

[15] NAVNIDHI, C. - BASHA, S. J.: Comparison of digital image watermarking methods DWT and DWTDCT on the basis of PSNR. International Journal of Innovative Research in Science, Engineering and Technology (IJIRSET), Vol. 1, No. 2, 2012.

[16] JIANSHENG, M. - SUKANG, L. - XIAOME, T.: A digital watermarking algorithm based on DCT and DWT. Proceedings of the 2009 International Symposium on Web Information Systems and Applications (WISA) China, pp. 104-107, 2009.
[17] REDDY, A. A. - CHATTERJI, B. N.: Anew wavelet based logo watermarking scheme. Conf. Pattern Recognition Letters, Vol. 26, No. 7, pp. 1019-1027, 2005.

[18] RAO, K. - DISCRETE, P. Y.: Cosine Transform: algorithms, advantages, applications. Academic Press, USA, 1990.

[19] XIN-YU, G. A. O. - JIAN-PING, L. V. J.: A blockbased DCT algorithm of digital image watermarking. Journal of Xi'an University of Posts and Telecommunications, Vol. 12, No. 5, 2007.

[20] GUANG-SHUAI, Z. - ZHIHAI, L. - GENLIN, J. PING, S. - JIAN-FEI, Y. - YUDONG, Z.: Automated Classification of Brain MR Images by WaveletEnergy and k-Nearest Neighbors Algorithm. 2015 Seventh International Symposium on Parallel Architectures, Algorithms and Programming, 2015.

[21] Watson, A. B.: DCT quantization matrice visually optimized for individual image. In Pro SPIE Conf. Human Vision, Visual Processing and Digital Display, London, 2000.

[22] Tsai, M. - Hung, H.: DCT and DWT based Image Watermarking Using Subsampling. In Proc. of the 2005 IEEE Fourth Int. Conf. on Machine Learning and Cybernetics, China, pp. 5308-5313, 2005.

[23] AL-HAJ, A.: Combined DWT-DCT Digital Image Watermarking. Journal of Computer Science, Vol. 3, No. 9, pp. 740-746, 2007.

[24] EJIMA, M. - MYAZAKI, A.: On the evaluation of performance of digital watermarking in the frequency domain. In Proc. of the IEEE Int. Conf. on Image Processing, Vol. 2, pp. 546-549, 2001.

[25] JIANG, J. - ARMSTRONG, A.: Data hiding approach for efficient image indexing. Electronics letters, Vol. 38, No. 23, pp. 1424-142, 2002.

Received August 07, 2018, accepted November 15, 2018

\section{BIOGRAPHIES}

Adegboye Mutiu Adesina is currently a Lecturer and researcher in the Department of Computer Engineering, Federal University, Oye-Ekiti, Nigeria. He obtained a Bachelor degree (B.Eng.) in Computer Engineering (2014) from Federal University of Technology,

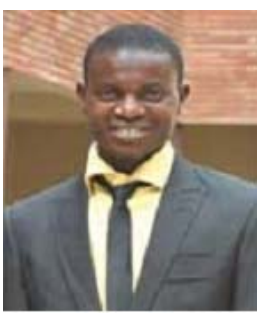

Minna, Niger state, Nigeria. He also obtained a Master degree (M.Eng) from the Department of Computer Engineering in the same institution. He is a graduate Register Engineer with the Council for the Regulations of Engineering in Nigeria (COREN) and also belongs to the following professional bodies; Professional member International Association of Engineers (IAENG), and Professional member of International Association of Computer Science and Information Technology (IASCIT). ADEGBOYE has published in conference proceeding and 
both national and international journals. His research interests are majorly in the areas of Computational Intelligence; Digital Signal Processing (DSP); Embedded system and Internet of Things (IoTs).

\section{Ajao Lukman Adewale is a Senior Technological Researcher in the Department of Computer Engineering, Federal University of Technology, Minna, Nigeria. He obtained Postgraduate Diploma in Computer Science (PGDCS) in 2013 from University of Ilorin, Ilorin,}

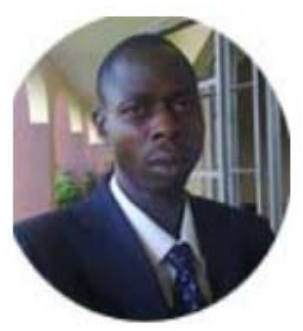
Nigeria. He has also obtained Master of Engineering (M.Eng) in the Department of Computer Engineering, Federal University of Technology, Minna. He is currently a $\mathrm{PhD}$ research student in the Department of Computer Engineering, Amadu Bello University, Zaira, Nigeria. His research interests are: Embedded System based Internet of Things, Wireless Sensor Network, FPGA and Networking.
Badmus Taofeeq Alabi is currently a Lecturer and researcher in the Department of Computer Engineering, Federal University, Oye-Ekiti, Nigeria. He obtained a Bachelor degree (B.Tech.) in the Department of Computer Science and Engineering (2011) with First Class Honour from Ladoke Akintola University of Technology Ogbomoso, Oyo State, Nigeria. He has obtained a Master degree (M.Tech) in the same department from Ladoke Akintola University of Technology Ogbomos, Oyo State, Nigeria. He is a graduate registered member of the Nigeria Society of Engineers (NSE) and also belongs to the following professional bodies; International Association of Engineers (IAENG), and a Professional member of International Association of Computer Science and Information Technology (IASCIT). BADMUS has published in conference proceeding and reputable scholarly international journals. His research interests is in System Modelling and Analysis; Digital Signal Processing (DSP); Intelligent Systems and their Applications 